\title{
The relationship between systemic inflammation, body composition and clinical outcomes in patients with operable colorectal cancer at low and medium to high nutritional risk
}

\author{
Tanvir Abbass* (D), Ross D. Dolan (D), Paul G. Horgan (D) \& Donald C. McMillan (D) \\ Academic Unit of Surgery, School of Medicine, University of Glasgow, Glasgow Royal Infirmary, Glasgow, G4 OSF, UK
}

\begin{abstract}
Background In accordance with European Society of Parenteral and Enteral Nutrition guidelines, the combination of malnutrition universal screening tool (MUST), systemic inflammation [modified Glasgow prognostic score (mGPS)] and body composition [skeletal muscle index (SMI) and skeletal muscle density (SMD)] were examined in relation to clinical outcomes in patients undergoing surgery for colorectal cancer (CRC).

Methods Data were collected for stages I-III CRC patients from prospectively maintained data base at the academic department of surgery, Glasgow Royal Infirmary. From the initial sample of 1046, pre-admission MUST score was available in 984 patients. The classification into low malnutrition risk (MUST $=0, n=810$ ) and moderate to high malnutrition risk (MUST 1 to $\geq 2$, $n=174$ ) groups and their relationship to systemic inflammatory response and body composition (SMI and SMD) with clinical outcomes were examined using univariate and multivariate analyses.

Results Compared with those patients at low nutrition risk (MUST $=0$ ), patients at moderate to high malnutrition risk (MUST 1 to $\geq 2)$ had an elevated mGPS $(P<0.001)$, neutrophil lymphocyte ratio (NLR) $(P<0.001)$, low SMI $(P \leq 0.001)$ and low SMD $(P=0.015)$. MUST was an important prognostic factor for length of hospital stay $(P<0.001)$ and 3 years overall survival $(P<0.001)$.

In low malnutrition risk patients (MUST $=0$ ), those who were systemically inflammed ( $m G P S 1 / 2, n=187$ ), had an elevated NLR $(P<0.001)$, low SMI $(P<0.001)$, low SMD $(P<0.01)$, increased post-operative complications $(P<0.05)$, longer hospital stay $>7$ days $(P<0.001)$, and poorer 3 years survival $(P<0.05)$ compared with those who were not systemically inflamed. On multivariate analysis, American Society of Anaesthesiologist (ASA) score $(P<0.05)$ and mGPS $(P<0.05)$ were independently associated with increased risk of clinical complications. ASA, mGPS, and NLR were independently associated with prolonged hospital stay $(P<0.05, P<0.05$, and $P<0.001$, respectively). ASA, tumour, node, metastasis stage, and mGPS were independently associated with overall survival $(P<0.01, P<0.001$, and $P<0.05$, respectively).

In medium-risk to high-risk patients (MUST $=1 / 2$ ), those who were systemically inflamed ( $m G P S 1 / 2, n=75$ ) had higher ASA $(P<0.05)$, elevated NLR $(P<0.01)$, low SMI $(P=0.05)$ and low SMD $(P<0.05)$, increased length of hospital stay $(P<0.05)$, and poorer 3 years survival $(P<0.01)$, compared with those who were not systemically inflamed.

Conlusions A small proportion of patients with primary operable CRC was at nutrition risk as defined by MUST alone in both low risk nutrition patients and medium/high risk nutrition patients. The systemic inflammatory response was associated with lower SMI, lower SMD, and poor clinical outcomes. The systemic inflammatory response is an important measure in the nutritional assessment of patients undergoing surgery for CRC.
\end{abstract}


Keywords Colorectal cancer (CRC); Malnutrition universal screening tool (MUST); Systemic inflammatory response (SIR); Modified Glasgow prognostic score (mGPS); Body composition; Skeletal muscle index (SMI); Skeletal muscle density (SMD)

Received: 6 March 2020; Revised: 30 May 2020; Accepted: 1 June 2020

*Correspondence to: Tanvir Abbass, Clinical Research Fellow, Academic Unit of Surgery, School of Medicine, University of Glasgow, Glasgow Royal Infirmary, Glasgow G4 OSF, United Kingdom. Phone: 0141211 4000, Fax: 0141211 4943, Email: tanvir.abbass@glasgow.ac.uk

\section{Introduction}

Cancer is a leading cause of death worldwide. Colorectal cancer (CRC) is the third most common cancer and is the fourth leading cause of cancer related deaths worldwide. Incidence of CRC is expected to increase by $60 \%$ to more than 2.2 million new cases and 1.1 million deaths by $2030 .^{1}$ In the UK, CRC is the common cancer with around 42, 000 new cases diagnosed annually and is the second most common cause of cancer death. ${ }^{2}$ Despite improvements in tumour staging and use of the multidisciplinary team based approach, post-operative complications and mortality persist, leading to poorer survival. Therefore, staging the tumour and staging the host are important steps in moving forward in the treatment of CRC. ${ }^{3}$

Malnutrition plays an important role in patients undergoing surgery for CRC. Various nutritional assessment methods are routinely used and, in the UK, malnutrition universal screening tool (MUST) has been adopted by the British Association of Parenteral and Enteral Nutrition. ${ }^{4}$ MUST score is a five-step process to categorize patients in low, medium, and high nutritional risk groups as shown in Figure 1 and in accordance with international nutritional guidelines. ${ }^{5}$ MUST score is widely used in National Health Service in the UK, and nursing and medical staff are familiar with its use and is included in the admission checklist. Its use in patients with cancer has been validated. ${ }^{6}$

Systemic inflammation measured by modified Glasgow prognostic score (mGPS) and neutrophil lymphocyte ratio (NLR) have been shown to have prognostic value in patients undergoing surgery for CRC. ${ }^{7}$ Recently, in a cohort of 363 patients undergoing surgery for CRC, it was reported that MUST was directly associated with MGPS and NLR and a low skeletal muscle index (SMI) measured using computed tomography (CT). ${ }^{8}$ However, it was of interest that approximately $80 \%$ of patients were at low nutritional risk, as defined by MUST, and in these patients, approximately $20 \%$ were systemically inflamed, and approximately $45 \%$ had a low SMI. Given that systemic inflammation and low SMI are directly related, ${ }^{9}$ it was of interest to examine their relationship and prognostic value in patients at low and medium to high nutrition risk.

The aim of the present study was to examine the relationship between malnutrition, systemic inflammation, body composition, and clinical outcomes in patients with operable CRC at low and medium to high nutritional risk.

Figure 1 The malnutrition universal screening tool (MUST). ${ }^{4}$ BMI, body mass index.

Points

MUST Score
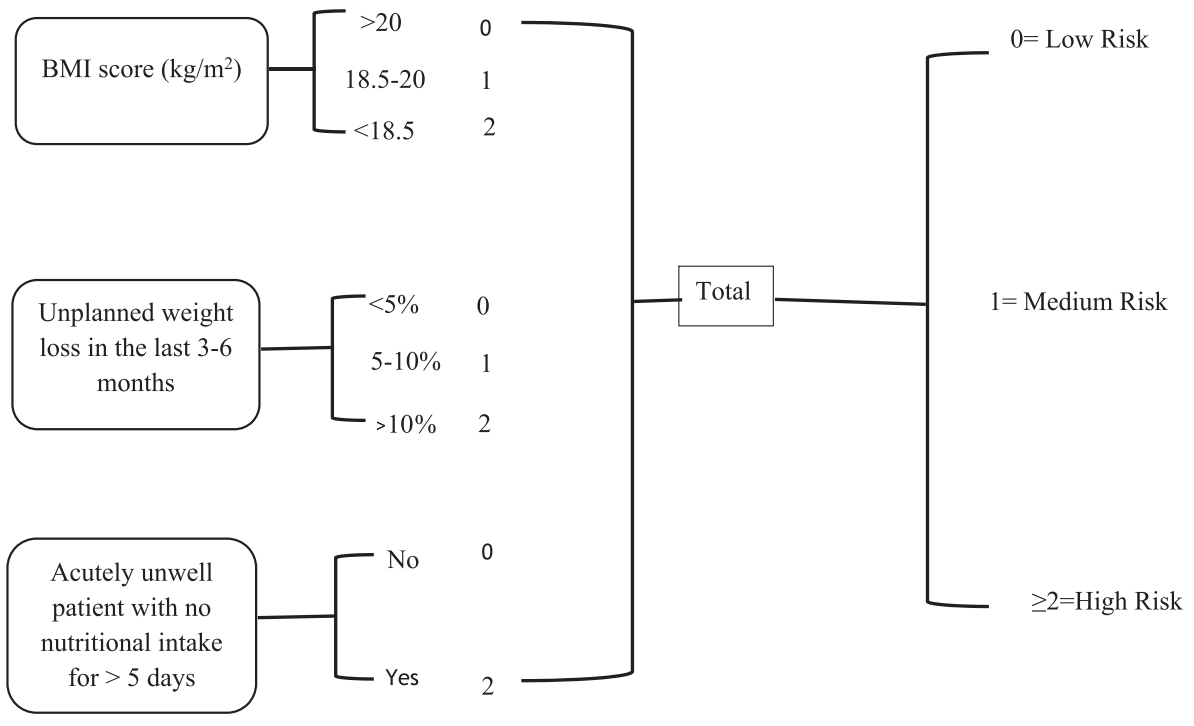


\section{Patients and methods}

\section{Patients}

Data were collected from prospectively maintained CRC data base at the academic department of surgery, Glasgow Royal Infirmary from March 2008 to March 2018. The flow diagram of included patients is shown in Figure 2. From an initial sample of 1060 patients, patients with stage IV disease and non-CRCs were excluded giving 1002 with stages I to III operable CRC patients. 984 patients had MUST scores available in this cohort, and these patients were entered into the study.

\section{Methods}

MUST score was calculated from pre-treatment admission record, and the relationship between MUST, clinico-pathological characteristics, systemic inflammation, body composition, and clinical outcomes in patients with primary operable CRC is shown in Table 1. Systemic inflammation was measured using
mGPS and NLR. Pre-operative mGPS and NLR were analysed from serum samples performed prior to surgery.

Body composition was assessed from the staging CT scan, and L3 DICOM images were retrieved from picture archiving and communication system (PACS). Body composition was analysed using image J software (https://imagej.nih.gov/ij/) by applying validated thresholds for CRC patients. ${ }^{10-12}$ Established thresholds of -29 to 150 Hounsfield unit (HU) for skeletal muscle and -190 to $-30 \mathrm{HU}$ for adipose tissue as previously described. ${ }^{13}$

The CT scans were analysed for total fat area (TFA), visceral fat area (VFA), subcutaneous fat area (SFA), skeletal muscle area (SMA), and skeletal muscle density (SMD). Measurements were performed by two individuals (TA and RD) on a sample of 40 patients. The interrater reliability was assessed using interclass correlation coefficients (ICCS). The ICC values were as follows: TFA and VFA $=0.999 ;$ SMA $=0.996$ and SMD $=0.993$. The cross-sectional area of fat and muscles was normalized for height squared $\left(\mathrm{m}^{2}\right)$ to calculate fat and skeletal muscle indices.

Subcutaneous adiposity was defined as SFI $>50 \mathrm{~cm}^{2} / \mathrm{m}^{2}$ in male patients and $>42 \mathrm{~cm}^{2} / \mathrm{m}^{2}$ in female patients. ${ }^{10}$ Visceral

Figure 2 Flow diagram of included patients with operable colorectal cancer. MUST, malnutrition universal screening tool.

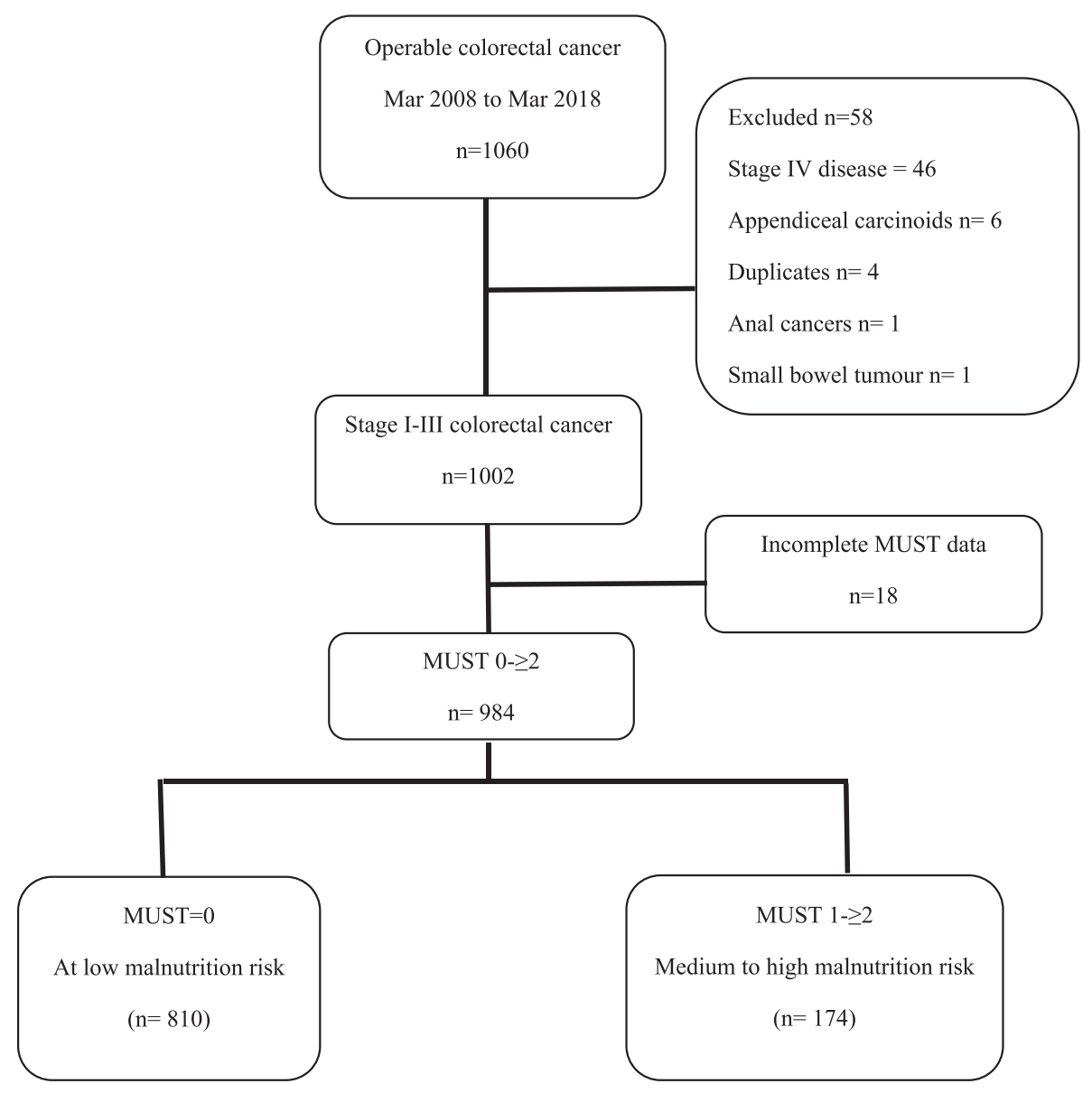


Table 1 The relationship between MUST, clinico-pathological characteristics, systemic inflammation, body composition, and clinical outcomes in patients with primary operable colorectal cancer

\begin{tabular}{|c|c|c|c|c|}
\hline & $\begin{array}{c}\text { Total } \\
n=984\end{array}$ & $\begin{array}{l}\text { MUST }=0 \\
n=810 \\
(82.3 \%)\end{array}$ & $\begin{array}{l}\text { MUST } 1 \text { to } \geq 2 \\
n=174 \\
(17.7 \%)\end{array}$ & $P$ value \\
\hline \multicolumn{5}{|l|}{ Clinico-pathological } \\
\hline Age (years) & & & & $<0.001$ \\
\hline$<65$ & $342(34.8)$ & $298(36.8)$ & $44(25.3)$ & \\
\hline $65-74$ & $362(36.8)$ & 309 (38.1) & $53(30.1)$ & \\
\hline$>74$ & $280(28.5)$ & $203(25.1)$ & 77 (44.3) & \\
\hline Sex & & & & 0.017 \\
\hline Male & $544(55.3)$ & $462(57)$ & $82(47.1)$ & \\
\hline Female & $440(44.7)$ & $348(43)$ & $92(52.9)$ & \\
\hline ASA & & & & 0.005 \\
\hline 1 & $193(19.6)$ & $167(20.6)$ & $26(14.9)$ & \\
\hline II & $450(45.7)$ & $374(46.1)$ & 76 (43.7) & \\
\hline III & $308(31.3)$ & 248 (30.6) & $60(34.5)$ & \\
\hline IV & $33(3.4)$ & $21(2.6)$ & $12(6.9)$ & \\
\hline Presentation & & & & $<0.001$ \\
\hline Elective & $922(93.7)$ & $770(95.1)$ & $152(87.4)$ & \\
\hline Emergency & $62(6.3)$ & $40(4.9)$ & $22(12.6)$ & \\
\hline TNM & & & & 0.140 \\
\hline 1 & 235 (23.9) & $204(25.2)$ & $31(17.8)$ & \\
\hline 2 & $398(40.4)$ & 320 (39.5) & $78(44.8)$ & \\
\hline 3 & $351(35.7)$ & $286(35.3)$ & $65(37.4)$ & \\
\hline Primary cancer & & & & 0.006 \\
\hline Colon & $588(59.8)$ & $468(57.8)$ & $120(69)$ & \\
\hline Rectum & $396(40.2)$ & $342(42.2)$ & $54(31)$ & \\
\hline $\begin{array}{l}\text { Adjuvant } \\
\text { treatment }\end{array}$ & & & & 0.245 \\
\hline No & $464(47.2)$ & $375(46.3)$ & $89(51.1)$ & \\
\hline Yes & $520(52.8)$ & $435(53.7)$ & 85 (48.9) & \\
\hline \\
\hline $\begin{array}{l}\text { inflammation } \\
\text { mGPS }\end{array}$ & & & & $<0.001$ \\
\hline 0 & $722(73.4)$ & $623(76.9)$ & $99(56.9)$ & \\
\hline 1 & $107(10.9)$ & $93(11.5)$ & $14(8)$ & \\
\hline 2 & $155(15.8)$ & $94(11.6)$ & $61(35.1)$ & \\
\hline NLR & & & & $<0.001$ \\
\hline$<3$ & $515(52.3)$ & $451(55.7)$ & $64(36.8)$ & \\
\hline $3-5$ & $303(30.8)$ & $236(29.1)$ & $67(38.5)$ & \\
\hline$>5$ & $166(16.9)$ & $123(15.2)$ & $43(24.7)$ & \\
\hline \multicolumn{5}{|c|}{ Body composition } \\
\hline $\begin{array}{l}\text { Subcutaneous } \\
\text { adiposity (Ebadi } \\
\text { threshold) }\end{array}$ & & & & $<0.001$ \\
\hline No & $197(20)$ & $122(15.1)$ & $75(43.1)$ & \\
\hline Yes & $787(80)$ & 688 (84.9) & 99 (56.9) & \\
\hline $\begin{array}{l}\text { Visceral obesity } \\
\text { (Doyle } \\
\text { threshold) }{ }^{11}\end{array}$ & & & & $<0.001$ \\
\hline No & $267(27.1)$ & $171(21.1)$ & $96(55.2)$ & \\
\hline Yes & 717 (72.9) & 639 (78.9) & 78 (44.8) & \\
\hline $\begin{array}{l}\text { Low SMI } \\
\text { (Martin } \\
\text { threshold) }{ }^{12}\end{array}$ & & & & $<0.001$ \\
\hline No & 491 (49.9) & $433(53.5)$ & $58(33.3)$ & \\
\hline Yes & $493(50.1)$ & 377 (46.5) & $116(66.7)$ & \\
\hline $\begin{array}{l}\text { Low SMD } \\
\text { (Martin } \\
\text { threshold) }{ }^{12}\end{array}$ & & & & 0.015 \\
\hline No & $\begin{array}{l}326(33.1) \\
658(66.9)\end{array}$ & $\begin{array}{l}282(34.8) \\
528(65.2)\end{array}$ & $\begin{array}{r}44(25.3) \\
130(74.7)\end{array}$ & \\
\hline $\begin{array}{l}\text { Yes } \\
\text { Postoperative } \\
\text { outcome }\end{array}$ & & & & \\
\hline
\end{tabular}

(Continues)
Table 1 (continued)

\begin{tabular}{|c|c|c|c|c|}
\hline & $\begin{array}{c}\text { Total } \\
n=984\end{array}$ & $\begin{array}{l}\text { MUST }=0 \\
n=810 \\
(82.3 \%)\end{array}$ & $\begin{array}{l}\text { MUST } 1 \text { to } \geq 2 \\
n=174 \\
(17.7 \%)\end{array}$ & $P$ value \\
\hline $\begin{array}{l}\text { Any } \\
\text { complications }\end{array}$ & & & & 0.410 \\
\hline No & $604(61.4)$ & $502(62)$ & $102(58.6)$ & \\
\hline Yes & $380(38.6)$ & 308 (38) & $72(41.4)$ & \\
\hline $\begin{array}{l}\text { Infective } \\
\text { complication }\end{array}$ & & & & 0.500 \\
\hline No & $738(75)$ & $611(75.4)$ & $127(73)$ & \\
\hline Yes & $246(25)$ & $199(24.6)$ & $47(27)$ & \\
\hline $\begin{array}{l}\text { Clavien-Dindo } \\
\text { grade }\end{array}$ & & & & 0.145 \\
\hline 0 & $604(61.4)$ & $502(62)$ & $102(58.6)$ & \\
\hline $1-2$ & $288(29.3)$ & $239(29.5)$ & 49 (28.2) & \\
\hline $3-5$ & $92(9.3)$ & $69(8.5)$ & $23(13.2)$ & \\
\hline $\begin{array}{l}\text { Length of } \\
\text { hospital stay } \\
\text { (days) }\end{array}$ & & & & $<0.001$ \\
\hline$\leq 7$ & $448(45.5)$ & $394(48.6)$ & $54(31)$ & \\
\hline$>7$ & $536(54.5)$ & 416 (51.4) & $120(69)$ & \\
\hline $\begin{array}{l}\text { Survival } \\
3 \text { year survival } \\
\% \text { (SE) }\end{array}$ & $85(1)$ & $90(1)$ & $67(4)$ & $<0.001$ \\
\hline
\end{tabular}

ASA, American Society of Anaesthesiologists; mGPS, modified Glasgow prognostic score; MUST, malnutrition universal screening tool; NLR, neutrophil lymphocyte ratio; SE, standard error; SMD, skeletal muscle density; SMI, skeletal muscle index; TNM, tumour, node, and metastasis.

obesity was defined as VFA $>160 \mathrm{~cm}^{2}$ in male patients and $>80 \mathrm{~cm}^{2}$ in female patients. ${ }^{11}$ Sarcopenia (low SMI) was defined per Martin threshold as SMI $<43 \mathrm{~cm}^{2} / \mathrm{m}^{2}$ in $\mathrm{BMI}<25 \mathrm{~kg} / \mathrm{m}^{2}$ and $\mathrm{SMI}<53 \mathrm{~cm}^{2} / \mathrm{m}^{2}$ in $\mathrm{BMI} \geq 25 \mathrm{~kg} / \mathrm{m}^{2}$ in male patients and $\mathrm{SMI}<41 \mathrm{~cm}^{2} / \mathrm{m}^{2}$ in $\mathrm{BMI}<25 \mathrm{~kg} / \mathrm{m}^{2}$ and $\mathrm{SMI}<41 \mathrm{~cm}^{2} / \mathrm{m}^{2}$ with BMI $\geq 25 \mathrm{~kg} / \mathrm{m}^{2}$ in female patients. Myosteatosis (low SMD) was defined as SMD $<41 \mathrm{HU}$ in patients with $\mathrm{BMI}<25 \mathrm{~kg} / \mathrm{m}^{2}$ and $\mathrm{SMD}<33 \mathrm{HU}$ in patients with $\mathrm{BMI} \geq 25 \mathrm{~kg} / \mathrm{m}^{2} .12$

\section{Statistical analysis}

$\chi^{2}$ test were used in the analysis of categorical variables. Logistic regression analysis was used to calculate odds ratio (OR) and $95 \%$ confidence intervals ( $\mathrm{Cls}$ ) for post-operative complications and length of hospital stay while cox proportional hazard model was used to calculate hazard ratio (HR) and $95 \% \mathrm{Cl}$ for overall survival. The Kaplan-Meier method and log rank test were used for survival analysis. $P$ value of $<0.05$ was considered significant.

The enrolled patients were followed up until death or 1 October 2019 whichever came first. Median duration of follow up was 52 months; three patients were lost to follow up. Post-operative complications within a month of surgery with 
their relationship to MUST were evaluated. Hospital stay was divided into $\leq 7$ days and $>7$ days as most of the patients undergoing CRC will be discharged by days 4 to 5 . Overall survival was defined as time in months from date of surgery to time of death from any cause or time to the end of study or loss to follow up. Patients were followed up as per CRC guidelines with tumour markers check every 3 months, CT follow up every 6 months and colonoscopy at 3 and 5 years post operatively for first 5 years. Of the 131 patients who died in this sample of 810 low nutritional risk patients, 115 died of CRC (16 other causes). All of the statistical analysis was performed using SPSS version 25 (IBM Corporation, 2017, Armonk, NY).

\section{Results}

In 1002 patients with stages I to III CRC, 984 had MUST scores available. Eight hundred ten patients (82\%) were at low nutritional risk (MUST $=0$ ), 174 patients $(18 \%)$ were at medium to high nutritional risk (MUST 1 to $\geq 2$ ). Mean age of patients was 68 years (range, 23-93). Fifty-five percent of patients were male $(n=544)$. The relationship between MUST and clinico-pathological factors is shown in Table 1. Compared with low MUST, moderate to high MUST was associated with older age $(P<0.001)$, female sex $(P<0.05)$, higher ASA $(P<0.01)$, emergency presentation $(P<0.001)$, colon cancer $(P<0.01)$, higher mGPS $(P<0.001)$ and NLR $(P<0.001)$, lower subcutaneous and visceral obesity (both $P<0.001$ ), low SMI $(P<0.001)$, low SMD $(P<0.05)$, longer hospital stay $(P<0.001)$ and poorer 3 year survival $(P<0.001)$.

In those patients at low nutrition risk ( $n=810,82 \%)$, the relationship between $\mathrm{mGPS}$ and clinico-pathological factors is shown in Table 2a. Compared with mGPS $0, \operatorname{mGPS~} 1 / 2$ was associated with higher ASA $(P<0.01)$, higher NLR $(P<0.001)$, low SMI $(P<0.001)$, low SMD $(P<0.01)$, greater length of hospital stay $(P<0.001)$, and poorer 3 year survival $(P<0.05)$.

In those patients at medium to high nutritional risk ( $n=174$, 18\%), the relationship between mGPS and clinico-pathological factors are shown in Table $2 \mathrm{~b}$. Compared with mGPS 0, mGPS $1 / 2$ was associated with higher ASA $(P<0.05)$, higher NLR $(P<0.01)$, low SMI $(P=0.05)$, low SMD $(P<0.05)$, longer hospital stay $(P<0.05)$, and poorer 3 years survival $(P<0.05)$.

The variables associated with post-operative complications in patients with operable CRC in low-risk MUST (MUST $=0$ ) are presented in Table 3. On univariate logistic regression, age $(P=0.014)$, sex $(P=0.052)$, ASA $(P=0.014)$, mGPS $(P=0.018)$, and NLR $(P=0.083)$ were significantly associated with post-operative complications. On multivariate analysis, age (OR 1.22; 95\% Cl: 1.02-1.48; $P=0.034$ ), ASA (OR 1.22; 95\% Cl: $1.01-1.47 ; P=0.035$ ), and mGPS (OR $1.25 ; 95 \% \mathrm{Cl}$ :
Table 2a The relationship between clinico-pathological characteristics, systemic inflammation, body composition, and clinical outcomes in patients with primary operable colorectal cancer at low nutritional risk patients (MUST $=0$ )

\begin{tabular}{llll}
\hline Total & (MUST $=0)$ & (MUST =0) & $P$ value \\
$n=810$ & with & with \\
& mGPS $=0$ & mGPS $=1 / 2$ \\
& $n=623$ & $n=187$ \\
& $(76.9 \%)$ & $(23.1 \%)$ \\
\hline
\end{tabular}

Clinico-pathological

Age (years)

$298(36.8) \quad 234(37.6)$

0.166

$65-74$

$309(38.1) \quad 241(38.7)$

$64(34.2)$

$>74$

Sex

Male

Female

$203(25.1) \quad 148(23.8)$

$68(36.4)$

$55(29.4)$

ASA

I

II

III

IV

Presentation

Elective

$462(57) \quad 354(56.8)$

$108(57.8)$

$79(42.2)$

$348(43) \quad 269(43.2)$

$167(20.6) \quad 134(21.5)$

$374(46.1) \quad 298(47.8)$

33 (17.6)

$76(40.6)$

$68(36.4)$

$\begin{array}{ccc}21(2.6) & 11(1.8) & 10(5.3)\end{array}$

Emergency

$770(95.1) \quad 596(95.7) \quad 174(93)$

TNM

1

$40(4.9) \quad 27(4.3)$

$13(7)$

$204(25.2) \quad 181(29.1)$

$23(12.3)$

$2320(39.5) 221(35.5)$

3

$286(35.3) \quad 221(35.5)$

Primary cancer

Colon

Rectum

$468(57.8) \quad 332(53.3)$

$342(42.2) \quad 291(46.7)$

$99(52.9)$

65 (34.8)

136 (72.7)

$51(27.3)$

Adjuvant

treatment

No $\quad 375(46.3) \quad 291(46.7) \quad 84(44.9)$

Yes $\quad 435(53.7) \quad 332(53.3) \quad 103(55.1)$

Systemic inflammation

NLR

$<3 \quad 451(55.7) \quad 376(60.4)$

3-5 $236(29.1) \quad 176(28.3)$

$>5 \quad 123(15.2) \quad 71(11.4)$

Body composition

Subcutaneous

adiposity (Ebadi

threshold) ${ }^{10}$

No

Yes

$122(15.1) \quad 92(14.8)$

$688(84.9) \quad 531(85.2)$

$30(16)$

Visceral obesity

(Doyle

threshold) $^{11}$

No

$171(21.1) \quad 131(21)$

Yes

639 (78.9) $492(79)$

$40(21.4)$

Low SMI (Martin

threshold) ${ }^{12}$

No $\quad 434(53.5) \quad 355(57) \quad 78(41.7)$

Yes $\quad 377(46.5) \quad 268(43) \quad 109(58.3)$

Low SMD (Martin

threshold) $^{12}$

$\begin{array}{llll}\text { No } & 282(34.8) & 234(37.6) & 48(25.7)\end{array}$

Yes $\quad 528(65.2) \quad 389(62.4) \quad 139(74.3)$

Clinical outcomes

Any complications

No

$501(62)$

398 (64)

$309(38)$

$225(36)$

$103(55.1)$

$84(44.9)$

Infective

complication

No

$610(75.3) \quad 476(76.4) \quad 134(71.7)$
0.821

0.004

0.148

0.013

$<0.001$

0.667

$<0.001$

0.669

0.915

$<0.001$

0.003

0.030

0.187 
Table 2a (continued)

\begin{tabular}{|c|c|c|c|c|}
\hline & $\begin{array}{ll}\text { Total } & ( \\
n=810 & \mathrm{v} \\
& n \\
& n\end{array}$ & $\begin{array}{l}(\text { MUST }=0) \\
\text { with } \\
\text { mGPS }=0 \\
n=623 \\
(76.9 \%)\end{array}$ & $\begin{array}{l}(\text { MUST }=0) \\
\text { with } \\
\text { mGPS }=1 / 2 \\
n=187 \\
(23.1 \%)\end{array}$ & $P$ value \\
\hline Yes & $200(24.7)$ & ) $147(23.6)$ & $53(28.3)$ & \\
\hline \multicolumn{4}{|l|}{$\begin{array}{l}\text { Clavien-Dindo } \\
\text { grade }\end{array}$} & 0.058 \\
\hline 0 & $501(61.9)$ & 398 (63.9) & $103(55.1)$ & \\
\hline $1-2$ & $240(29.6)$ & $174(27.9)$ & $66(35.3)$ & \\
\hline $3-5$ & $69(8.5)$ & $51(8.2)$ & $18(9.6)$ & \\
\hline \multicolumn{4}{|c|}{$\begin{array}{l}\text { Length of hospital } \\
\text { stay (days) }\end{array}$} & $<0.001$ \\
\hline$\leq 7$ & 394 (48.6) & $324(52)$ & $70(37.4)$ & \\
\hline$>7$ & 416 (51.4) & $299(48)$ & $117(62.6)$ & \\
\hline \multirow{2}{*}{\multicolumn{2}{|c|}{$\begin{array}{l}\text { Survival } \\
3 \text { year survival } \% 90 \% \text { (1) } \\
\text { (SE) }\end{array}$}} & & & 0.048 \\
\hline & & $91 \%(1)$ & $87 \%(3)$ & \\
\hline
\end{tabular}

1.01-1.54; $P=0.037$ ) were independently associated with post-operative complications.

The variables associated with length of hospital stay in patients with operable CRC in low-risk MUST (MUST =0) are presented in Table 4. On univariate logistic regression analysis, age $(P=0.001)$, ASA $(P<0.001)$, tumour, node, metastasis (TNM) stage $(P=0.019)$, mGPS $(P<0.001)$, NLR $(P<0.001)$, and low SMI $(P=0.010)$ were significantly associated with prolonged hospital stay $>7$ days. On multivariate analysis, ASA (OR 1.28; 95\% Cl: 1.05-1.56; $P=0.014$ ), mGPS (OR 1.30; 95\% Cl: 1.04-1.63; $P=0.022$ ), and NLR (OR 1.65; 95\% $\mathrm{Cl}: 1.35-2.03 ; P<0.001)$ were independently associated with prolonged hospital stay $>7$ days.

The variables associated with overall survival in patients with operable CRC in low-risk MUST (MUST =0) are shown in Table 5. A total of 679 patients (84\%) were alive at censor date in MUST = 0 group. Death due to any cause occurred in 131 (16\%) patients. The median survival was 58 months (range 0-139 months). After exclusion of 30 days mortality, six patients $(0.7 \%)$, there was significant association between MUST score and overall survival $(P<0.001)$.

On univariate Cox regression survival analysis, age $(P<0.001)$, sex $(P=0.021)$, ASA $(P<0.001)$, TNM stage $(P<0.001)$, mGPS $(P=0.003)$, NLR $(P=0.023)$, low SMI $(P=0.076)$, and low SMD $(P=0.005)$ were significantly associated with overall survival. On multivariate analysis, age (HR 1.41; 95\% Cl: 1.21-1.79; $P=0.004)$, sex (HR $1.49 ; 95 \% \mathrm{Cl}: 1.04-2.15 ; p=0.031)$, ASA (HR 1.44; 95\% Cl:1.13-1.83; $p=0.003$ ), TNM stage (HR 1.80; 95\% Cl:1.40-2.31; $p<0.001$ ), mGPS (HR 1.29; 95\% Cl: $1.03-$ $1.62 ; p=0.026$ ) were independently associated with overall survival.
Table $\mathbf{2 b}$ The relationship between clinico-pathological characteristics, systemic inflammation, body composition, and clinical outcomes in patients with primary operable colorectal cancer at medium to high risk of malnutrition (MUST 1 to $\geq 2$ )

\begin{tabular}{|c|c|c|c|c|}
\hline & $\begin{array}{ll}\text { Total } & n \\
n=174 \geq & \\
& n \\
& n\end{array}$ & $\begin{array}{l}\text { MUST } 1 \text { to } \\
\geq 2 \text { with } \\
\text { mGPS }=0 \\
n=99 \\
(56.9 \%)\end{array}$ & $\begin{array}{c}\text { MUST } 1 \text { to } \\
\geq 2 \text { with } \\
\text { mGPS }=1 / 2 \\
n=75 \\
(43.1 \%)\end{array}$ & $\begin{array}{l}P \\
\text { value }\end{array}$ \\
\hline \multicolumn{5}{|l|}{ Clinico-pathological } \\
\hline Age (years) & & & & 0.676 \\
\hline$<65$ & $44(25.3)$ & $24(24.2)$ & $20(26.7)$ & \\
\hline $65-74$ & $53(30.5)$ & $30(30.3)$ & $23(30.7)$ & \\
\hline$>74$ & $77(44.3)$ & $45(45.5)$ & $32(42.7)$ & \\
\hline \multicolumn{4}{|c|}{ 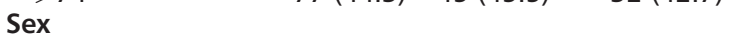 } & 0.155 \\
\hline Male & $82(47.1)$ & $42(42.4)$ & $40(53.3)$ & \\
\hline Female & 92 (52.9) & $57(57.6)$ & $35(46.7)$ & \\
\hline ASA & & & & 0.024 \\
\hline I & 26 (14.9) & $22(22.2)$ & $4(5.3)$ & \\
\hline II & $76(43.7)$ & $40(40.4)$ & $36(48)$ & \\
\hline III & $60(34.5)$ & $31(31.3)$ & $29(38.7)$ & \\
\hline IV & $12(6.9)$ & $6(6.1)$ & $6(8)$ & \\
\hline \multicolumn{4}{|l|}{ Presentation } & 0.248 \\
\hline Elective & $152(87.4)$ & $89(89.9)$ & $63(84)$ & \\
\hline Emergency & $22(12.6)$ & $10(10.1)$ & $12(16)$ & \\
\hline TNM & & & & 0.075 \\
\hline 1 & $31(17.8)$ & $26(26.3)$ & $5(6.7)$ & \\
\hline 2 & $78(44.8)$ & $36(36.4)$ & $42(56)$ & \\
\hline 3 & $65(37.4)$ & $37(37.4)$ & $28(37.3)$ & \\
\hline \multicolumn{4}{|l|}{ Primary cancer } & 0.002 \\
\hline Colon & $120(69)$ & 59 (59.6) & $61(81.3)$ & \\
\hline Rectum & $54(31)$ & $40(40.4)$ & $14(18.7)$ & \\
\hline \multicolumn{4}{|c|}{ Adjuvant treatment } & 0.102 \\
\hline No & $89(51.1)$ & $56(56.6)$ & $33(44)$ & \\
\hline Yes & 85 (48.9) & $43(43.4)$ & $42(56)$ & \\
\hline \multicolumn{5}{|l|}{$\begin{array}{l}\text { Systemic } \\
\text { inflammation }\end{array}$} \\
\hline NLR & & & & 0.006 \\
\hline$<3$ & $64(36.8)$ & $45(45.5)$ & $19(25.3)$ & \\
\hline $3-5$ & $67(38.5)$ & $35(35.4)$ & $32(42.7)$ & \\
\hline$>5$ & $43(24.7)$ & 19 (19.2) & $24(32)$ & \\
\hline \\
\hline \multicolumn{4}{|l|}{$\begin{array}{l}\text { Subcutaneous } \\
\text { adiposity } \\
\text { threshold) }\end{array}$} & 0.473 \\
\hline No & $75(43.1)$ & $45(45.5)$ & $30(40)$ & \\
\hline Yes & 99 (56.9) & $54(54.5)$ & $45(60)$ & \\
\hline \multicolumn{4}{|l|}{$\begin{array}{l}\text { Visceral obesity } \\
\text { (Doyle threshold) }\end{array}$} & 0.300 \\
\hline No & $96(55.2)$ & $58(58.6)$ & $38(50.7)$ & \\
\hline Yes & $78(44.8)$ & $41(41.4)$ & $37(49.3)$ & \\
\hline \multicolumn{4}{|l|}{$\begin{array}{l}\text { Low SMI (Martin } \\
\text { threshold) }\end{array}$} & 0.052 \\
\hline No & $58(33.3)$ & 39 (39.4) & $19(25.3)$ & \\
\hline Yes & $116(66.7)$ & $60(60.6)$ & $56(74.7)$ & \\
\hline \multicolumn{4}{|l|}{$\begin{array}{l}\text { Low SMD (Martin } \\
\text { threshold) }{ }^{12}\end{array}$} & 0.036 \\
\hline No & $44(25.3)$ & $31(31.3)$ & $13(17.3)$ & \\
\hline Yes & $130(74.7)$ & 68 & $62(82.7)$ & \\
\hline \multicolumn{5}{|l|}{$\begin{array}{l}\text { Postoperative } \\
\text { outcome }\end{array}$} \\
\hline \multicolumn{4}{|l|}{ Any complications } & 0.358 \\
\hline No & $102(58.6)$ & $61(61.6)$ & $41(54.7)$ & \\
\hline Yes & $72(41.4)$ & 38 (38.4) & $34(45.3)$ & \\
\hline \multicolumn{4}{|l|}{$\begin{array}{l}\text { Infective } \\
\text { complication }\end{array}$} & 0.003 \\
\hline No & $127(73)$ & $81(81.8)$ & $46(61.3)$ & \\
\hline
\end{tabular}

(Continues) 
Table $2 \mathrm{~b}$ (continued)

\begin{tabular}{|c|c|c|c|c|}
\hline & $\begin{array}{ll}\text { Total } & \\
n=174 & \geq \\
& r \\
& n\end{array}$ & $\begin{array}{l}\text { MUST } 1 \text { to } \\
\geq 2 \text { with } \\
\text { mGPS }=0 \\
n=99 \\
(56.9 \%)\end{array}$ & $\begin{array}{c}\text { MUST } 1 \text { to } \\
\geq 2 \text { with } \\
\text { mGPS }=1 / 2 \\
n=75 \\
(43.1 \%)\end{array}$ & $\begin{array}{l}P \\
\text { value }\end{array}$ \\
\hline Yes & $47(27)$ & $18(18.2)$ & $29(38.7)$ & \\
\hline Clavien-Dindo grade & & & & 0.515 \\
\hline 0 & $102(58.6)$ & ) $61(61.6)$ & $41(54.7)$ & \\
\hline $1-2$ & $49(28.2)$ & $25(25.3)$ & $24(32)$ & \\
\hline $3-5$ & $23(13.2)$ & ) $13(13.1)$ & $10(13.3)$ & \\
\hline $\begin{array}{l}\text { Length of hospita } \\
\text { stay (days) }\end{array}$ & & & & 0.038 \\
\hline$\leq 7$ & $54(31)$ & $37(37.4)$ & $17(22.7)$ & \\
\hline$>7$ & $120(69)$ & $62(62.6)$ & $58(77.3)$ & \\
\hline $\begin{array}{l}\text { Survival } \\
3 \text { year survival } \% \\
\text { (SE) }\end{array}$ & \% $67(4)$ & 73 (5) & $58(6)$ & 0.003 \\
\hline
\end{tabular}

ASA, American Society of Anaesthesiologists score; mGPS, modified Glasgow prognostic score; MUST, malnutrition universal screening tool; NLR, neutrophil lymphocyte ratio; SMD, skeletal muscle density; SMI, skeletal muscle index; TNM, tumour, node, and metastasis.

\section{Discussion}

The results of the present study in almost 1000 patients with primary operable CRC show that approximately $80 \%$ of patients was at low nutritional risk. In those patients, at low nutrition risk (MUST 0), the systemic inflammatory response, as evidenced by an elevated mGPS $1 / 2$, was associated with low SMI, greater length of hospital stay, and poorer overall survival. Similarly, in those patients at moderate/high nutritional risk (MUST 1/2), an elevated mGPS was associated with low SMI, greater length of hospital stay, and poorer overall survival. Therefore, the combined assessment of MUST and
mGPS has complementary value and may form the basis of a routine disease-related malnutrition assessment in patients with primary operable CRC.

The MUST tool is simple to use and provides recommendation for healthcare professionals to improve nutritional status of patients. MUST has been validated in terms of predicting clinical outcomes and compares favourably with other nutritional risk assessments. ${ }^{14,15}$ Similarly, the mGPS is simple and objective to use, has been extensively validated in predicting clinical outcomes, and compares favourably with other systemic inflammation-based prognostic scores. ${ }^{7,16}$ Therefore, the combined use in cancer patients undergoing nutritional assessment is worthy of further study.

The results of the present study are consistent with the recommendations of a recent task force commissioned by four major international clinical nutrition societies: European Society of Parenteral and Enteral Nutrition (ESPEN), American Society of Parenteral and Enteral Nutrition (ASPEN), Parenteral and Enteral Nutrition Society of Asia (PENSA) and Latin American Federation for Parenteral and Enteral Nutrition (FELANPE). They proposed that the diagnosis of malnutrition was based on three phenotypic criteria (unintentional weight loss, low body mass index, and low muscle mass) and two etiologic criteria (low food intake or low food assimilation and inflammation or disease burden) and that to diagnose malnutrition at least one phenotypic and one etiologic criteria should be present. Therefore, when malnutrition is caused by an underlying chronic disease, it may be termed disease-related malnutrition and in the presence of a systemic inflammatory response may be considered to be cachexia. $^{17}$

In the present study of patients with operable CRC, there was no direct measure of physical function made and so we were

Table 3 The relationship between clinico-pathological characteristics, systemic inflammation, body composition and any post-operative complications patients with primary operable colorectal cancer, at low risk MUST (MUST $=0$ )

\begin{tabular}{|c|c|c|c|c|}
\hline \multirow[t]{2}{*}{ Variables } & Univariate analysis & \multirow[t]{2}{*}{$P$ value } & Multivariate analysis & \multirow[t]{2}{*}{$P$ value } \\
\hline & OR $(95 \% \mathrm{Cl})$ & & OR $(95 \% \mathrm{Cl})$ & \\
\hline \multicolumn{5}{|l|}{ Clinico-pathological } \\
\hline Age $(<65 / 65-74 />74)$ & $1.27(1.05-1.52)$ & 0.014 & $1.22(1.02-1.48)$ & 0.034 \\
\hline Sex (Male/Female) & $1.33(1.00-1.78)$ & 0.052 & $1.31(0.98-1.74)$ & 0.073 \\
\hline ASA (I-IV) & $1.26(1.05-1.52)$ & 0.014 & $1.22(1.01-1.47)$ & 0.035 \\
\hline TNM stage (I-III) & $1.08(0.90-1.30)$ & 0.408 & - & - \\
\hline \multicolumn{5}{|l|}{ Systemic inflammation } \\
\hline mGPS $(0 / 1 / 2)$ & $1.28(1.04-1.57)$ & 0.018 & $1.25(1.01-1.54)$ & 0.037 \\
\hline $\operatorname{NLR}(<3 / 3-5 />5)$ & $1.18(0.98-1.43)$ & 0.083 & & 0.303 \\
\hline \multicolumn{5}{|l|}{ Body Composition } \\
\hline Subcutaneous adiposity (Ebadi threshold) ${ }^{10}$ & $1.15(0.77-1.72)$ & 0.493 & - & - \\
\hline Visceral adiposity (Doyle threshold) ${ }^{11}$ & $1.14(0.80-1.61)$ & 0.476 & - & - \\
\hline Low SMI (Martin threshold) ${ }^{12}$ & $1.10(0.83-1.47)$ & 0.500 & - & - \\
\hline Low SMD (Martin threshold) $^{12}$ & $1.08(0.80-1.45)$ & 0.624 & - & - \\
\hline
\end{tabular}

Binary logistic regression variables with $P<0.1$ on univariate analysis were entered into backward conditional multivariate analysis. $P$ value $<0.05$ was considered significant. ASA, American Society of Anaesthesiologists; $\mathrm{Cl}$, confidence interval; mGPS, modified Glasgow prognostic score; NLR, neutrophil lymphocyte ratio; OR, odds ratio; SMD, skeletal muscle radiodensity; SMI, skeletal muscle index; TNM, tumour, node, metastasis. 
Table 4 The relationship between clinico-pathological characteristics, systemic inflammation, body composition, and length of hospital stay $(\leq 7$ or $>7$ days) in patients with primary operable colorectal cancer, at low nutrition risk (MUST $=0$ )

\begin{tabular}{|c|c|c|c|c|}
\hline \multirow[t]{2}{*}{ Variables } & Univariate analysis & \multirow[t]{2}{*}{$P$ value } & Multivariate analysis & \multirow[t]{2}{*}{$P$ value } \\
\hline & OR $(95 \% \mathrm{Cl})$ & & OR $(95 \% \mathrm{Cl})$ & \\
\hline \multicolumn{5}{|l|}{ Clinico-pathological } \\
\hline Age $(<65 / 65-74 />74)$ & $1.36(1.14-1.63)$ & 0.001 & $1.21(1.00-1.47)$ & 0.055 \\
\hline Sex (Male/Female) & $1.12(0.85-1.48)$ & 0.416 & - & - \\
\hline ASA (I-IV) & $1.42(1.19-1.71)$ & $<0.001$ & $1.28(1.05-1.56)$ & 0.014 \\
\hline TNM stage (I-III) & $1.24(1.04-1.49)$ & 0.019 & $1.19(0.98-1.43)$ & 0.074 \\
\hline \multicolumn{5}{|l|}{ Systemic inflammation } \\
\hline mGPS $(0 / 1 / 2)$ & $1.54(1.24-1.91)$ & $<0.001$ & $1.30(1.04-1.63)$ & 0.022 \\
\hline $\operatorname{NLR}(<3 / 3-5 />5)$ & $1.78(1.47-2.17)$ & $<0.001$ & $1.65(1.35-2.03)$ & $<0.001$ \\
\hline \multicolumn{5}{|l|}{ Body composition } \\
\hline Subcutaneous adiposity (Ebadi threshold) ${ }^{10}$ & $1.03(0.70-1.50)$ & 0.897 & - & - \\
\hline Visceral adiposity (Doyle threshold) $^{11}$ & $1.15(0.82-1.62)$ & 0.406 & - & - \\
\hline Low SMI (Martin threshold) ${ }^{12}$ & $1.44(1.09-1.90)$ & 0.010 & - & 0.200 \\
\hline Low SMD (Martin threshold) ${ }^{12}$ & $1.32(0.99-1.77)$ & 0.059 & - & 0.965 \\
\hline
\end{tabular}

Binary logistic regression variables with $P<0.1$ on univariate analysis were entered into backward conditional multivariate analysis. $P$ value $<0.05$ was considered significant. ASA, American Society of Anaesthesiologists; Cl, confidence interval; mGPS, modified Glasgow prognostic score; NLR, neutrophil lymphocyte ratio; OR, odds ratio; SMD, skeletal muscle radiodensity; SMI, skeletal muscle index; TNM, tumour, node, metastasis.

unable to explore the relationship between the systemic inflammatory response and physical activity in these patients. However, in a recent publication, the relationship between the MGPS and measures of physical activity was examined in patients with advanced lung cancer. ${ }^{18}$ This study showed that an elevated mGPS was significantly and independently associated with poorer handgrip strength. Therefore, there is some evidence that the presence of the systemic inflammatory response is associated with a poor capacity for activity in patients with cancer.

The strengths of the present study include a large sample size and detailed phenotypic characterization of patients with primary operable CRC. However, there are a number of limitations. The present study is from a single institution and therefore may not be representative of all patients with primary operable CRC. Also, the MUST-defined cohort of moderate/high risk patients was relatively small. Therefore, confirmatory studies are required. However, given the simplicity of our study approach, it is likely that this work will be readily repeated.

It will be interesting to longitudinally study this relationship between MUST, systemic inflammation, and body composition in CRC cohort. Improving fitness of patient by addressing malnutrition with muscle mass and function coupled with decrease in stress of surgery (inflammatory response) will help in reducing adverse post-operative outcome and achieve the best possible outcome for patient. Reducing catabolism and improving anabolic response by addressing nutrition, inflammation, muscle mass, and function are important components in treatment of patients with CRC.

Table 5 The relationship between clinico-pathological characteristics, systemic inflammation, body composition, and overall survival in patients with primary operable colorectal cancer, at low nutritional risk (MUST =0)

\begin{tabular}{|c|c|c|c|c|}
\hline \multirow[t]{2}{*}{ Variables } & Univariate analysis & \multirow[t]{2}{*}{$P$ value } & Multivariate analysis & \multirow[t]{2}{*}{$P$ value } \\
\hline & $\mathrm{HR}(95 \% \mathrm{Cl})$ & & $\mathrm{HR}(95 \% \mathrm{Cl})$ & \\
\hline \multicolumn{5}{|l|}{ Clinico-pathological } \\
\hline Age $(<65 / 65-74 />74)$ & 1.59 (1.27-1.99) & $<0.001$ & $1.41(1.21-1.79)$ & 0.004 \\
\hline Sex (Male/Female) & $1.52(1.06-2.18)$ & 0.021 & $1.49(1.04-2.15)$ & 0.031 \\
\hline ASA (I-IV) & $1.67(1.33-2.09)$ & $<0.001$ & $1.44(1.13-1.83)$ & 0.003 \\
\hline TNM stage (I-III) & $1.80(1.41-2.29)$ & $<0.001$ & $1.80(1.40-2.31)$ & $<0.001$ \\
\hline \multicolumn{5}{|l|}{ Systemic inflammation } \\
\hline mGPS $(0 / 1 / 2)$ & $1.43(1.15-1.79)$ & 0.003 & $1.29(1.03-1.62)$ & 0.026 \\
\hline $\operatorname{NLR}(<3 / 3-5 />5)$ & $1.30(1.04-1.62)$ & 0.023 & & 0.275 \\
\hline \multicolumn{5}{|l|}{ Body composition } \\
\hline Subcutaneous adiposity (Ebadi threshold) ${ }^{10}$ & $0.85(0.54-1.35)$ & 0.505 & - & - \\
\hline Visceral adiposity (Doyle threshold) ${ }^{11}$ & $1.05(0.69-1.60)$ & 0.821 & - & - \\
\hline Low SMI (Martin threshold) ${ }^{12}$ & $1.36(0.97-1.92)$ & 0.076 & & 0.859 \\
\hline Low SMD (Martin threshold) $^{12}$ & $1.69(1.15-2.48)$ & 0.005 & & 0.235 \\
\hline
\end{tabular}

Cox regression analysis variables with $P<0.1$ on univariate analysis were entered into backward conditional multivariate analysis. $P$ value $<0.05$ was considered significant. ASA, American Society of Anaesthesiologists; Cl, confidence interval; HR, hazard ratio; mGPS, modified Glasgow Prognostic Score; NLR, neutrophil lymphocyte ratio; SMD, skeletal muscle radiodensity; SMI, skeletal muscle index; TNM, tumour, node, metastasis. 


\section{Conclusions}

The combination of MUST and MGPS would appear to provide a reliable objective assessment tool for risk stratification of length of hospital stay and survival in patients with primary operable CRC.

\section{Funding}

The authors declare no financial support or funding received for this study purposes.

\section{Conflict of interest}

The authors declare no potential conflicts of interest.

\section{Author contribution}

TA is responsible for the conceptualization, data curation, data analysis, and writing the original and final draft versions. $\mathrm{RD}$ performed data analysis and validation of results. $\mathrm{PH}$ reviewed the article. DCM carried out the conceptualization, reviewing, and editing. All authors have read and approved the manuscript.

\section{Disclaimer}

The authors certify that they comply with the ethical guidelines for publishing in the Journal of Cachexia, Sarcopenia and Muscle. ${ }^{19}$

\section{References}

1. Bray F, Ferlay J, Soerjomataram I, Siegel RL, Torre LA, Jemal A. Global cancer statistics 2018: GLOBOCAN estimates of incidence and mortality worldwide for 36 cancers in 185 countries. CA Cancer J Clin 2018;68:394-424.

2. Cancer Research UK. Cancer statistics for UK. 2019.

3. Park JH, Ishizuka M, McSorley ST, Kubota K, Roxburgh CSD, Nagata $\mathrm{H}$, et al. Staging the tumour and staging the host: a two centre, two country comparison of systemic inflammatory responses of patients undergoing resection of primary operable colorectal cancer. Am J Surg 2018;216:458-464.

4. Elia M. Screening for malnutrition: a multidisciplinary responsibility. Development and use of the 'Malnutrition Universa Screening Tool' ('MUST') for adults. BAPEN 2003.

5. Arends J, Baracos V, Bertz H, Bozzetti F, Calder PC, Deutz NEP, et al. ESPEN expert group recommendations for action against cancer-related malnutrition. Clin Nutr (Edinburgh, Scotland) 2017;36:1187-1196.

6. Boleo-Tome C, Monteiro-Grillo I, Camilo M, Ravasco P. Validation of the malnutrition universal screening tool (MUST) in cancer. Br J Nutr 2012;108:343-348.

7. Dolan RD, Lim J, McSorley ST, Horgan PG, McMillan DC. The role of the systemic inflammatory response in predicting outcomes in patients with operable cancer: systematic review and meta-analysis. Sci Rep 2017;7:16717.

8. Almasaudi AS, McSorley ST, Dolan RD, Edwards CA, McMillan DC. The relation between malnutrition universal screening tool (MUST), computed tomography-derived body composition, systemic inflammation, and clinical outcomes in patients undergoing surgery for colorectal cancer. Am J Clin Nutr 2019;110:1327-1334.

9. Abbass T, Dolan RD, Laird BJ, McMillan DC The relationship between imaging-based body composition analysis and the systemic inflammatory response in patients with cancer: a systematic review. Cancers (Basel) 2019;11:1304.

10. Ebadi M, Martin L, Ghosh S, Field CJ Lehner R, Baracos VE, et al. Subcutaneous adiposity is an independent predictor of mortality in cancer patients. $\mathrm{Br} J$ Cancer 2017;117:148-155.

11. Doyle SL, Bennett AM, Donohoe $\mathrm{CL}$, Mongan AM, Howard JM, Lithander FE, et al. Establishing computed tomography-defined visceral fat area thresholds for use in obesity-related cancer research. Nutr Res 2013;33:171-179.

12. Martin L, Birdsell L, Macdonald N, Reiman $\mathrm{T}$, Clandinin MT, McCargar $\amalg$, et al. Cancer cachexia in the age of obesity: skeletal muscle depletion is a powerful prognostic factor, independent of body mass index. $J$ Clin Oncol Off J Am Soc Clin Oncol 2013;31:1539-1547.

13. Dolan RD, Almasaudi AS, Dieu LB, Horgan PG, McSorley ST, McMillan DC. The relationship between computed tomography-derived body composition, systemic inflammatory response, and survival in patients undergoing surgery for colorectal cancer. J Cachexia Sarcopenia Muscle 2019;10:111-122.

14. Henderson S, Moore N, Lee E, Witham MD. Do the malnutrition universal screening tool (MUST) and Birmingham nutrition risk (BNR) score predict mortality in older hospitalised patients? BMC Geriatr 2008;8:26

15. Stratton RJ, King CL, Stroud MA, Jackson AA, Elia M. 'Malnutrition Universal Screening Tool' predicts mortality and length of hospital stay in acutely ill elderly. Br J Nutr 2006;95:325-330.

16. Dolan RD, McSorley ST, Horgan PG, Laird B, McMillan DC. The role of the systemic inflammatory response in predicting outcomes in patients with advanced inoperable cancer: systematic review and meta-analysis. Crit Rev Oncol Hemat 2017;116:134-146.

17. Muscaritoli M, Arends J, Aapro M. From guidelines to clinical practice: a roadmap for oncologists for nutrition therapy for cancer patients. Ther Adv Med Oncol 2019;11:1758835919880084.

18. Dolan RD, Daly LE, Simmons CP, Ryan AM, Sim WM, Fallon $M$, et al. The relationship between ECOG-PS, mGPS, BMI/WL grade and body composition and physical function in patients with advanced cancer. Cancers (Basel) 2020;12:1187.

19. von Haehling S, Morley JE, Coats AJS, Anker SD. Ethical guidelines for publishing in the Journal of Cachexia, Sarcopenia and Muscle: update 2017. J Cachexia Sarcopenia Muscle 2017;8:1081-1083. 